\title{
DIFFRACTED TRANSITIVE RADIATION AS A MEANS FOR INDICATING THE DIVERGENCE OF AN ULTRARELATIVISTIC ELECTRON BEAM
}

\begin{abstract}
S. V. Blazhevich, ${ }^{1}$ M. V. Bronnikova, ${ }^{1}$ and A. V. Noskov ${ }^{2}$
UDC 537.8

Diffracted transition radiation (DTR) emitted by a beam of relativistic electrons traversing a thin single-crystal plate in the Laue scattering geometry is considered. An expression has been obtained describing the angular DTR density when the electron path length in a target is far less than the extinction length of $x$-rays in the crystal. It is shown that in this case, the DTR process has a clearly pronounced kinematic character. Numerical calculations of the DTR photon yield in the direction of Bragg's scattering performed for different solid angles of recording show that it is significantly affected by the electron beam divergence. We have concluded that the DTR photon yield measured for a given solid angle can be used for indicating the electron beam divergence. Results of model calculations of the electron beam divergence parameters for a given yield of DTR photons traversing the slit collimator have shown that the formula proposed in this work can be successfully used as a basis for the development of methods for measuring the divergence of ultrahigh-energy relativistic electron beams based on the angular DTR distribution.
\end{abstract}

Keywords: relativistic electrons, diffracted transition radiation, indicating the electron beam divergence.

\section{INTRODUCTION}

When carrying out fundamental and applied experimental investigations of high-energy electron beams, the researchers face the problem of insufficient information on the parameters of the employed beams. The transverse sizes and the divergence are the key parameters of the beams. The main problem for physicists dealing with the electron beams having energies in the range $100-1000 \mathrm{MeV}$ is measuring the transverse beam sizes, because the beam divergence of modern accelerators (of the order of $0.001 \mathrm{mrad}$ ) is insignificant for the beams with transverse sizes of the order of or greater than $10 \mu \mathrm{m}$. Nowadays two new electron-positron colliders are being developed [1, 2]. In these colliders, electrons and positrons will be accelerated to energy of $250 \mathrm{GeV}$ in beams with very small transverse sizes $(\sim 5-100 \mathrm{~nm})$, and the main problem here will be the measurement of the beam divergence. A solution of this problem will allow experimental data of both fundamental and applied research to be interpreted more precisely. In [3, 4] the feasibility of application of relativistic electrons of parametric x-ray radiation (PXR) for diagnostics of the transverse relativistic beam sizes was experimentally investigated.

The influence of the electron beam divergence on PXR in a crystal was experimentally investigated in Tomsk and Tokyo [5] for electrons with energies of 600 and $800 \mathrm{MeV}$, respectively. The researchers showed that the orientational dependence of PXR generated by relativistic electrons in a crystal was sensitive to the beam divergence and proposed to use PXR as a simple means for determining the angular divergence of beams of high-energy charged particles. In [6] it was proposed to use the parametric x-ray radiation generated in thin crystals to obtain real-time data

${ }^{1}$ National Research University "Belgorod State University,” Belgorod, Russia, e-mail: blazh@bsu.edu.ru; mvb12@mail.ru; ${ }^{2}$ Belgorod State Technological University named after V. G. Shukhov, Belgorod, Russia, e-mail: noskovburk@mail.ru. Translated from Izvestiya Vysshikh Uchebnykh Zavedenii, Fizika, No. 6, pp. 100-112, June, 2020. Original article submitted January 9, 2020. 
on the electron beam location and sizes. PXR in the direction of Bragg's scattering is accompanied by diffracted transition radiation (DTR) [7-10] generated on the front side of a crystal target. We have developed the dynamic theory of coherent $\mathrm{x}$-ray radiation excited by diverging beams of relativistic electrons traversing a single-crystal plate in the Laue scattering geometry for the general case of electron field reflection asymmetric relative to the target surface [11].

Expressions for the angular density of coherent radiation obtained in [11] showed that DTR photons emitted by beams of electrons with energies exceeding several hundred megaelectronvolts have a much narrower angular distribution than the PXR photons. As a result, the angular DTR density is more sensitive to the beam divergence. With further increase in the relativistic electron energy, the angular distribution of the generated DTR photons becomes narrower, while the width of the angular distribution of PXR photons saturates and ceases to change. In this case, the maximum of the angular PXR density is located far outside the range of DTR angles at which its intensity becomes many times lower than the DTR intensity. Thus, as the electron energy increased, the only suitable means for indicating the parameters of electron beams with the relativistic factor $\gamma>2000$ remains DTR. For the effective DTR application for this purpose, it is important to determine the most suitable characteristics of its angular distribution. In particular, it is possible to expect that the use of the integral DTR characteristics will considerably simplify the indication process. In the present work, we demonstrate the feasibility of application of the integral DTR photon yield measured within a preset solid angle (the collimated DTR photon yield) for this purpose. Diffracted transition radiation of ultrarelativistic electrons in a single crystal is studied in the Laue scattering geometry. The case of a very thin target is considered when multiple scattering of electrons on atoms of the target is insignificant. It is an important case, because it provides measurements with very small distortions of the measurable parameters. An expression describing the angular DTR density is obtained when the electron path length in the target is much less than the extinction length. It is shown that in this case, the expression obtained has a clearly pronounced kinematic character. It should be noted that DTR for such a small target thickness has not yet been considered. Conventionally, DTR was considered only for the case when the electron path length was much greater than the extinction length, that is, when the DTR waves in a single crystal are dynamically diffracted [7-11]. In this regard, the formulas used in [7-11] are dynamic.

In the present work, we investigate the feasibility of application of the integral yield of photons of collimated DTR emitted by an ultrarelativistic electron beam traversing a very thin single crystal target to analyze the beam divergence. We assume that under these conditions, the PXR contribution to coherent radiation is negligibly small; therefore, we will neglect it.

\section{GEOMETRY OF THE RADIATIVE PROCESS}

Let us consider a beam of relativistic electrons traversing a crystal plate (Fig. 1). The interaction of each electron in the beam with the target is considered to be independent; therefore, the spectral-angular density of radiation generated by the electron beam can be obtained by averaging of the expression for the spectral-angular density of radiation generated by a single electron of the beam over all possible electron trajectories in the target.

For this purpose, we have introduced the angular variable $\boldsymbol{\psi}$ that relates the electron velocity vector $\boldsymbol{V}$ (expressed in units of the velocity of light in free space) to the unit vector of the $\boldsymbol{e}_{1}$ axis of the electron beam into the formula for the spectral-angular DTR density [11]. We have also introduced the angular variable $\boldsymbol{\theta}_{0}$ relating the unit vector in the direction of the incident pseudo-photon $\boldsymbol{n}$ of the Coulomb electron field to the vector $\boldsymbol{e}_{1}$ and the angular variable $\boldsymbol{\theta}$ relating the unit vector in the direction of photon diffraction $\boldsymbol{n}_{g}$ to the unit vector $\boldsymbol{e}_{2}$ (defined by the Bragg ratios $\boldsymbol{e}_{1} \boldsymbol{\theta}_{0}=0$ and $\boldsymbol{e}_{1} \boldsymbol{e}_{2}=\cos 2 \theta_{\mathrm{B}}$ ). These relationships for ultrarelativistic electrons and small values of the angular variables, describing the angular distributions of electrons and photons, have the following forms [11]

$$
\boldsymbol{V}=\left(1-\frac{1}{2} \gamma^{-2}-\frac{1}{2} \psi^{2}\right) \boldsymbol{e}_{1}+\boldsymbol{\psi}, \boldsymbol{e}_{1} \boldsymbol{\psi}=0,
$$




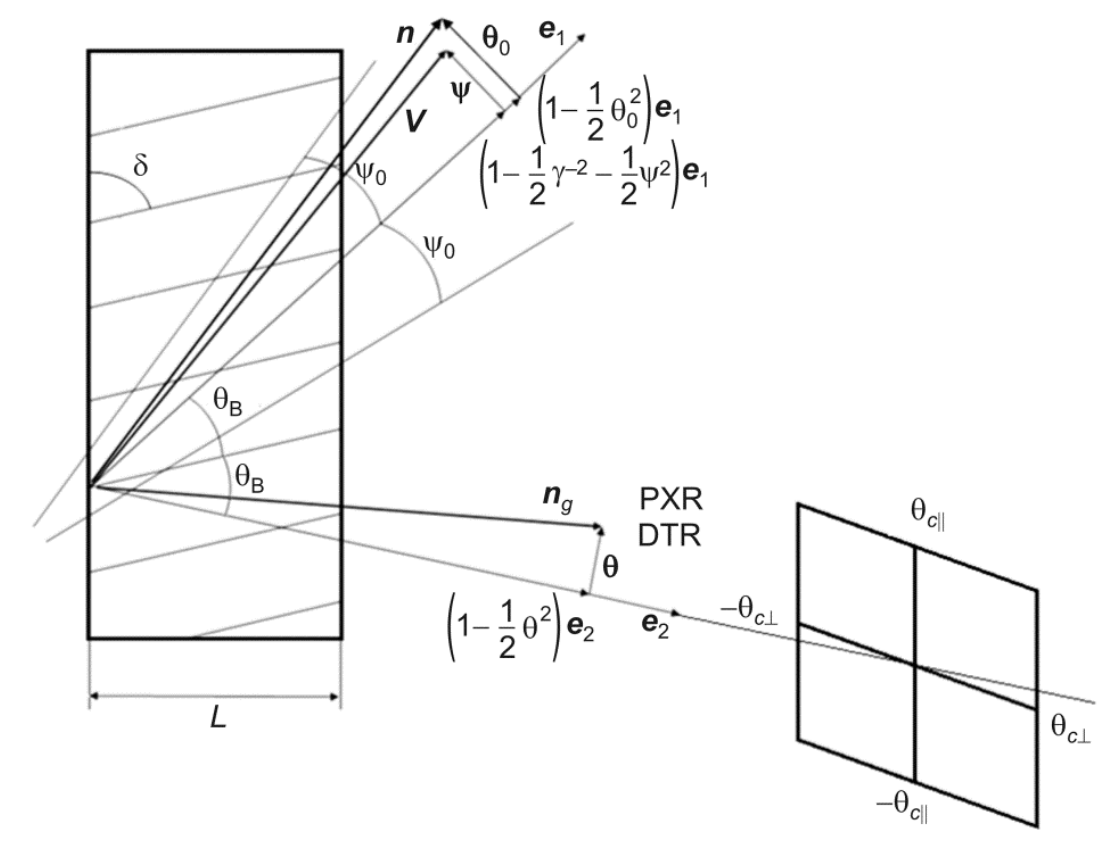

Fig. 1. Geometry of the radiative process. The solid angle of DTR photon recording is $\Delta \Omega=4\left(\theta_{c \|} \cdot \theta_{c \perp}\right)$, where $\theta_{c \|}$ and $\theta_{c \perp}$ are the maximum collimator angles with respect to the collimator center located on the $\boldsymbol{e}_{2}$ axis (see the integration limits in formula (11)).

$$
\begin{gathered}
\boldsymbol{n}=\left(1-\frac{1}{2} \theta_{0}^{2}\right) \boldsymbol{e}_{1}+\boldsymbol{\theta}_{0}, \boldsymbol{e}_{1} \boldsymbol{\theta}_{0}=0, \boldsymbol{e}_{1} \boldsymbol{e}_{2}=\cos 2 \theta_{\mathrm{B}}, \\
\boldsymbol{n}_{g}=\left(1-\frac{1}{2} \theta^{2}\right) \boldsymbol{e}_{2}+\boldsymbol{\theta}, \boldsymbol{e}_{2} \boldsymbol{\theta}=0,
\end{gathered}
$$

where $\gamma=1 / \sqrt{1-V^{2}}$ is the Lorentz factor of the particle and $\theta_{\mathrm{B}}$ is Bragg's scattering angle. The angular vector variables have components in the parallel and perpendicular planes: $\boldsymbol{\theta}=\boldsymbol{\theta}_{\|}+\boldsymbol{\theta}_{\perp}, \boldsymbol{\theta}_{0}=\boldsymbol{\theta}_{0 \|}+\boldsymbol{\theta}_{0 \perp}, \boldsymbol{\psi}=\boldsymbol{\psi}_{\|}+\boldsymbol{\psi}_{\perp}$ (see Fig. 1).

\section{SPECTRAL-ANGULAR DTR DENSITY IN A THIN SINGLE-CRYSTAL PLATE}

We take advantage of the formula obtained in [11] to describe the spectral-angular density of DTR emitted by a relativistic electron in a single crystal of arbitrary thickness $L$ :

$$
\omega \frac{d^{2} N_{\mathrm{DTR}}^{(s)}}{d \omega d \Omega}=\frac{e^{2}}{4 \pi^{2}} \Omega^{(s) 2}\left(\frac{1}{\gamma^{-2}+\left(\theta_{\perp}-\psi_{\perp}\right)^{2}+\left(\theta_{\|}+\psi_{\|}\right)^{2}}-\frac{1}{\gamma^{-2}+\left(\theta_{\perp}-\psi_{\perp}\right)^{2}+\left(\theta_{\|}+\psi_{\|}\right)^{2}-\chi_{0}^{\prime}}\right)^{2} R_{\mathrm{DTR}}^{(s)},
$$




$$
R_{\text {DTR }}^{(s)}=\frac{\varepsilon^{2}}{\xi(\omega)^{2}+\varepsilon}\left[\exp \left(-2 b^{(s)} \rho^{(s)} \Delta^{(1)}\right)+\exp \left(-2 b^{(s)} \rho^{(s)} \Delta^{(2)}\right)-2 \exp \left(-b^{(s)} \rho^{(s)} \frac{1+\varepsilon}{\varepsilon}\right) \cos \left(\frac{2 b^{(s)} \sqrt{\xi^{(s) 2}+\varepsilon}}{\varepsilon}\right)\right]
$$

where

$$
\begin{gathered}
\Omega^{(1)}=\theta_{\perp}-\psi_{\perp}, \Omega^{(2)}=\theta_{\|}+\psi_{\|}, \\
\Delta^{(2)}=\frac{\varepsilon+1}{2 \varepsilon}+\frac{1-\varepsilon}{2 \varepsilon} \frac{\xi^{(s)}}{\sqrt{\xi^{(s)^{2}}+\varepsilon}}+\frac{\kappa^{(s)}}{\sqrt{\xi^{(s)^{2}}+\varepsilon}}, \Delta^{(1)}=\frac{\varepsilon+1}{2 \varepsilon}-\frac{1-\varepsilon}{2 \varepsilon} \frac{\xi^{(s)}}{\sqrt{\xi^{(s)^{2}}+\varepsilon}}-\frac{\kappa^{(s)}}{\sqrt{\xi^{(s)^{2}}+\varepsilon}}, \varepsilon=\frac{\sin \left(\delta+\theta_{\mathrm{B}}\right)}{\sin \left(\delta-\theta_{\mathrm{B}}\right)}, \\
\rho^{(s)}=\frac{\chi_{0}^{\prime \prime}}{\left|\chi_{g}^{\prime}\right| C^{(s)}}, b^{(s)}=\frac{1}{2 \sin \left(\delta-\theta_{\mathrm{B}}\right)} \frac{L}{L_{\mathrm{ext}}^{(s)}}, v^{(s)}=\frac{\chi_{g}^{\prime} C^{(s)}}{\chi_{0}^{\prime}}, \kappa^{(s)}=\frac{\chi_{g}^{\prime \prime} C^{(s)}}{\chi_{0}^{\prime \prime}}, \xi^{(s)}(\omega)=\eta^{(s)}(\omega)+\frac{1-\varepsilon}{2 v^{(s)}}, \\
\eta^{(s)}(\omega)=\frac{2 \sin ^{2} \theta_{\mathrm{B}}}{V^{2}\left|\chi_{g}^{\prime}\right| C^{(s)}}\left(\frac{\omega\left(1-\theta_{\|} \cot \theta_{\mathrm{B}}\right)}{\omega_{\mathrm{B}}}-1\right), C^{(1)}=1, C^{(2)}=\cos 2 \theta_{\mathrm{B}}, d \Omega \equiv d \theta_{\|} \cdot d \theta_{\perp},
\end{gathered}
$$

$\chi_{g}=\chi_{g}^{\prime}+i \chi_{g}^{\prime \prime}$ is the coefficient of the Fourier series expansion of the dielectric susceptibility vector of the crystal in the reciprocal lattice vectors $\boldsymbol{g}$, and $\chi_{0}=\chi_{0}^{\prime}+i \chi_{0}^{\prime \prime}$ is the average dielectric susceptibility. We here used the Heaviside-Lorentz system of units.

At $s=1$, Eq. (2) describes $\sigma$-polarized waves, and at $s=2$, it describes $\pi$-polarized waves. Equation (2) describes the spectral-angular density of DTR of a relativistic electron traversing the single crystal plate and considers the angular deviation of the electron velocity vector (of the angle $\psi\left(\psi_{\perp}, \psi_{\|}\right)$) from the electron beam axis $\boldsymbol{e}_{1}$. This expression was derived within the limits of the two-wave approach of dynamical diffraction theory for the general case of asymmetric reflection of radiation waves. The asymmetry of the reflection for the fixed Bragg scattering angle is determined by the angle between the reflecting system of parallel atomic crystal planes and the target surface (by the angle $\delta$ ). The parameter $\varepsilon$ determines the degree of asymmetry of reflection of the Coulomb fields of the electron with respect to the target surface. We note that the angle $\left(\delta-\theta_{\mathrm{B}}\right)$ of the electron beam incidence on the target surface decreases with increasing parameter $\varepsilon$. The parameter $b^{(s)}$ is equal to half the electron path length in the target $L_{e}=L / \sin \left(\delta-\theta_{\mathrm{B}}\right)$, expressed in terms of the extinction length $L_{\mathrm{ext}}^{(s)}=1 / \omega\left|\chi_{\boldsymbol{g}}^{\prime}\right| C^{(s)}$.

We consider radiation excited by the relativistic electron beam in the thin non-absorbing single crystal plate, that is, when the maximum path length of the photon diffracted in the plate $L_{f}=L / \sin \left(\delta+\theta_{\mathrm{B}}\right)$ is much shorter than the absorption length $L_{\mathrm{abs}}=1 / \omega \chi_{0}^{\prime \prime}$ of x-ray waves in the crystal:

$$
2 \frac{b^{(s)} \rho^{(s)}}{\varepsilon}=\frac{L_{f}}{L_{\mathrm{abs}}}<<1
$$

It can be easily demonstrated that in this case, Eq. (2b) can be reduced to the form

$$
R_{\mathrm{DTR}}^{(s)}=\frac{4 \varepsilon^{2}}{\xi^{(s) 2}+\varepsilon} \sin ^{2}\left(b^{(s)} \frac{\sqrt{\xi^{(s) 2}+\varepsilon}}{\varepsilon}\right) .
$$


It can also be noted that the parameter $b^{(s)}$ can take values $b^{(s)}>>1$ even when inequality (4) is satisfied. To obtain the angular DTR density, we integrate Eq. (2) over the frequency $\omega$ using the formula $\frac{d \omega}{\omega}=\frac{\left|\chi_{g}^{\prime}\right| C^{(s)}}{2 \sin ^{2} \theta_{\mathrm{B}}} d \xi^{(s)}$ that follows from the expression for the function $\xi^{(s)}(\omega)$ in Eqs. (3). In this case, the angular DTR density assumes the following form:

$$
\frac{d N_{\mathrm{DTR}}^{(s)}}{d \Omega}=\frac{e^{2}}{8 \pi^{2}} \frac{\left|\chi_{g}^{\prime}\right| C^{(s)} \Omega^{(s) 2}}{\sin ^{2} \theta_{\mathrm{B}}}\left(\frac{1}{\gamma^{-2}+\left(\theta_{\perp}-\psi_{\perp}\right)^{2}+\left(\theta_{\|}+\psi_{\|}\right)^{2}}-\frac{1}{\gamma^{-2}+\left(\theta_{\perp}-\psi_{\perp}\right)^{2}+\left(\theta_{\|}+\psi_{\|}\right)^{2}-\chi_{0}^{\prime}}\right)^{2} \int_{-\infty}^{\infty} R_{\mathrm{DTR}}^{(s)} d \xi^{(s)},
$$

which can be re-written as

$$
\frac{d N_{\mathrm{DTR}}^{(s)}}{d \Omega}=\frac{e^{2} \chi_{0}^{\prime 2}\left|\chi_{g}^{\prime}\right| C^{(s)}}{8 \pi^{2} \sin ^{2} \theta_{\mathrm{B}}} \frac{\Omega^{(s) 2}}{\left(\gamma^{-2}+\left(\theta_{\perp}-\psi_{\perp}\right)^{2}+\left(\theta_{\|}+\psi_{\|}\right)^{2}-\chi_{0}^{\prime}\right)^{2}\left(\gamma^{-2}+\left(\theta_{\perp}-\psi_{\perp}\right)^{2}+\left(\theta_{\|}+\psi_{\|}\right)^{2}\right)^{2}} \int_{-\infty}^{\infty} R_{\mathrm{DTR}}^{(s)} d \xi^{(s)}(\omega) .
$$

For a very thin target, we assume that $b^{(s)}<<\sqrt{\varepsilon}$. Using the formula $\int_{-\infty}^{\infty} \frac{\sin ^{2}(x)}{x^{2}} d x=\pi$, integral (6) with the spectral function $R_{\mathrm{DTR}}^{(s)}$ given by Eq. (5) can be approximated as follows: $\int_{-\infty}^{\infty} R_{\mathrm{DTR}}^{(s)} d \xi^{(s)}(\omega) \approx 4 \pi \varepsilon b^{(s)}$. In this case, formula (6) for the angular DTR density assumes the form

$$
\frac{d N_{\mathrm{DTR}}^{(s)}}{d \Omega}=\frac{e^{2} \omega_{\mathrm{B}} \chi_{0}^{\prime 2} \chi_{g}^{\prime 2} C^{(s) 2}}{4 \pi \sin ^{2} \theta_{\mathrm{B}}} \frac{\Omega^{(s) 2}}{\left(\gamma^{-2}+\left(\theta_{\perp}-\psi_{\perp}\right)^{2}+\left(\theta_{\|}+\psi_{\|}\right)^{2}-\chi_{0}^{\prime}\right)^{2}\left(\gamma^{-2}+\left(\theta_{\perp}-\psi_{\perp}\right)^{2}+\left(\theta_{\|}+\psi_{\|}\right)^{2}\right)^{2}} \varepsilon \frac{L}{\sin \left(\delta-\theta_{\mathrm{B}}\right)} .
$$

If relativistic electrons have huge energy $\left(\gamma>>\frac{1}{\sqrt{\left|\chi_{0}^{\prime}\right|}}\right)$ and the modulus of the electron deviation angle in the beam $\psi\left(\psi_{\perp}, \psi_{\|}\right)$is less than or of the order of the characteristic angle corresponding to the maximum of the distribution $\operatorname{DTR}\left(\gamma^{-1}\right)$, that is, if $\gamma^{-2}+\left(\theta_{\perp}-\psi_{\perp}\right)^{2}+\left(\theta_{\|}+\psi_{\|}\right)^{2}<<-\chi_{0}^{\prime}$, Eq. (7) assumes the form

$$
\frac{d N_{\mathrm{DTR}}^{(s)}}{d \Omega}=\frac{e^{2} \omega_{\mathrm{B}} \chi_{g}^{\prime 2} C^{(s) 2}}{4 \pi \sin ^{2} \theta_{\mathrm{B}}} \frac{\Omega^{(s) 2}}{\left(\gamma^{-2}+\left(\theta_{\perp}-\psi_{\perp}\right)^{2}+\left(\theta_{\|}+\psi_{\|}\right)^{2}\right)^{2}} \varepsilon \frac{L}{\sin \left(\delta-\theta_{\mathrm{B}}\right)} .
$$

Formula (8) that describes the angular density of DTR generated by a high-energy electron in a thin single-crystal target is kinematic in character. This is indicated by the direct proportionality of the dependence of the angular density on the thickness $L$ of the single-crystal target. The condition $b^{(s)}<<\sqrt{\varepsilon}$ implies that the electron path length is much shorter than the extinction length of the x-ray waves in the crystal, which excludes the energy transfer from the incident wave to the diffracted wave. In this case, DTR is mainly formed when the electron approaches the target boundary in vacuum. In this case, electrons in the medium near the boundary are set into vibrational motion and emit electromagnetic waves that are constructively summed up in the direction of Bragg's scattering. Under these conditions, the dynamic reflection of transitive radiation from the system of parallel atomic planes of the crystal is absent. 


\section{INFLUENCE OF THE ELECTRON BEAM DIVERGENCE ON THE DTR YIELD}

Since DTR of ultrahigh-energy electrons has narrow angular distribution, it is expedient to consider the application of the dependence of the DTR photon yield normalized to one electron within a given solid angle near the direction of Bragg's scattering on the initial electron beam divergence. For this purpose, we performed averaging of the expressions for the number of emitted photons over all possible rectilinear trajectories of electrons in the beam. As an example, we performed the DTR averaging for the electron beam with Gaussian angular distribution:

$$
f(\psi)=\frac{1}{\pi \psi_{0}^{2}} e^{-\frac{\psi^{2}}{\psi_{0}^{2}}}
$$

where we call the parameter $\psi_{0}$ the emitted electron beam divergence (see Fig. 1). The angle $\psi_{0}$ defines the cone that covers the part of the electron beam out of which the electron flux density decreased more than $e$ times compared to the density on the beam axis.

For the number of DTR photons $\left\langle N_{\text {DTR }}\right\rangle$ emitted into the solid angle $4\left(\theta_{c \|} \cdot \theta_{c \perp}\right)$, we take advantage of the expression

$$
\left\langle N_{\mathrm{DTR}}\right\rangle=\frac{1}{\pi \psi_{0}^{2}} \int_{-\theta_{c \|}}^{\theta_{c \|}} \int_{\theta_{c \perp}}^{\theta_{c \perp}} \int_{-\infty}^{\infty} \int_{-\infty}^{\infty} \sum_{s=1}^{2} \frac{d N_{\mathrm{DTR}}^{(s)}}{d \Omega}\left(\psi_{\perp}, \psi_{\|}, \theta_{\perp}, \theta_{\|}\right) e^{-\frac{\psi_{\perp}{ }^{2}+\psi_{\|}{ }^{2}}{\psi_{0}^{2}}} d \psi_{\perp} d \psi_{\|} d \theta_{\perp} d \theta_{\|} .
$$

This expression contains summation of the angular DTR density over two projections of photon polarization, integration over all photon emission angles, and averaging over angular electron distribution (9) in the beam. Based on Eq. (8) for the angular DTR density, we derive the corresponding expression for the number of DTR photons $\left\langle N_{\text {DTR }}\right\rangle$ emitted by the ultrarelativistic electron beam from the thin single-crystal target into a preset angle near the direction of Bragg's scattering:

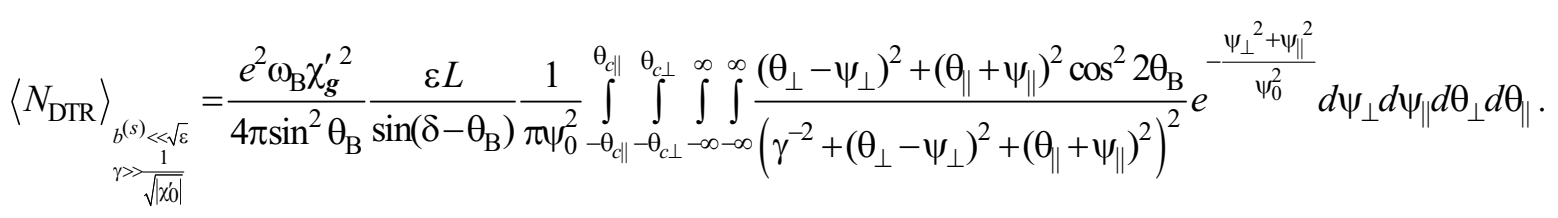

Figure 2 shows the dependence of the average number of the DTR photons (normalized to one incident photon) in the rectangular collimator with collimation angles $2 \theta_{c \perp}$ and $2 \theta_{c \|}$ emitted in the Bragg direction (axis $\boldsymbol{e}_{2}$ ) on the divergence $\psi_{0}$ of the electron beam incident on the crystal (see Fig. 1). The curves are shown for the indicated values of the solid angles of recording at $\theta_{c \perp}=\theta_{c \|}$. Calculations were performed for the (111)-oriented thin diamond target. The discrepancy between the solid curves and the curves shown by bold points reflects the violation of the condition $\gamma^{-2}+\left(\theta_{\perp}-\psi_{\perp}\right)^{2}+\left(\theta_{\|}+\psi_{\|}\right)^{2}<<-\chi_{0}^{\prime}$ in the case of application of Eq. (11) for angles in the range $\theta_{c \perp}=\theta_{c \|}>3 / \gamma$. From Fig. 2 it can be seen that the total DTR yield is practically independent of the beam divergence for a sufficiently large collimation angle. The optimal collimation should provide a maximгь dependence of the DTR yield on the divergence that corresponds to the maximum derivative $\left|d N_{\mathrm{DTR}} / d \psi_{0}\right|$. Figure 3 shows curves $1-4$ from Fig. 2 in an enlarged scale.

The influence of the DTR collimation on the resolution of measurements of the electron beam divergence is illustrated by Fig. 4 that shows the calculated dependence of the DTR photon yield derivative with respect to the beam 


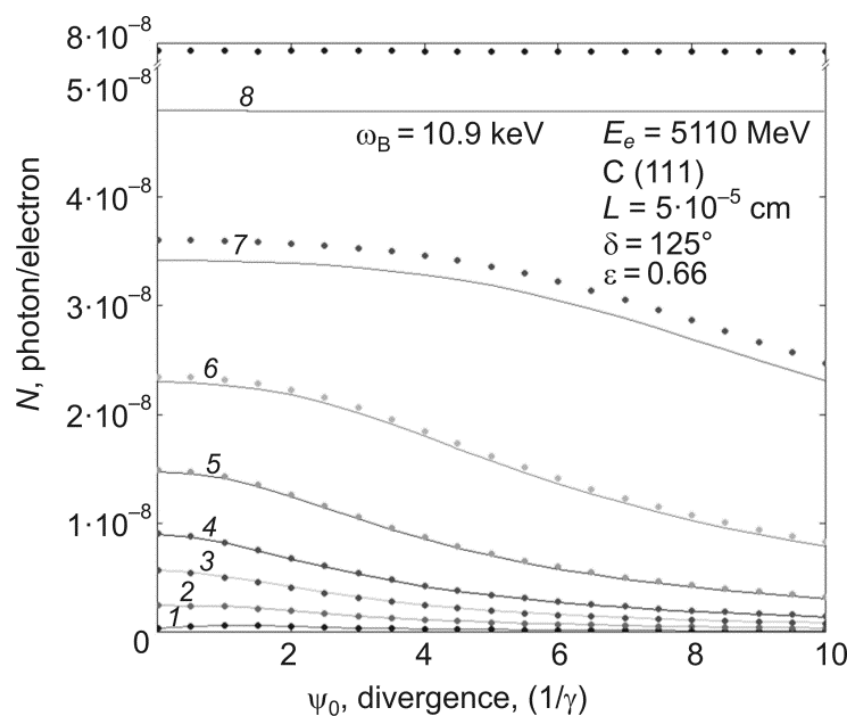

Fig. 2. Dependence of the DTR photon yield $N_{\mathrm{ph}}$ on the electron beam divergence $\psi_{0}$ calculated using exact formula (10) with the angular density given by Eq. (6) (the solid curves) and formula (11) (bold points) for the Lorentz factor of the electron $\gamma=10^{4}$ and the solid angle of recording $\Delta \Omega=4\left(\theta_{c \|} \cdot \theta_{c \perp}\right): \theta_{c \|}=\theta_{c \perp}=0.5 \gamma^{-1}$ (curve 1$), \gamma^{-1}$ (curve 2), $1.5 \gamma^{-1}$ (curve 3), $2 \gamma^{-1}$ (curve 4), $3 \gamma^{-1}$ (curve 5), $5 \gamma^{-1}$ (curve 6), $10 \gamma^{-1}$ (curve 7), and $100 \gamma^{-1}$ (curve 8 ).

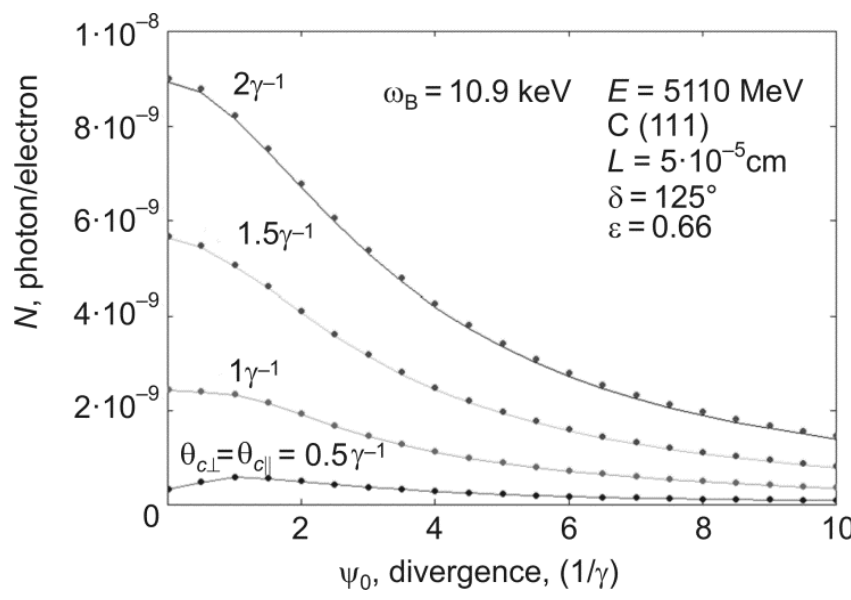

Fig. 3. Same as in Fig. 2, but in the enlarged scale.

divergence $\left|d N_{\text {DTR }} / d \psi_{0}\right|$. The family of curves in Fig. 4 illustrates the influence of the DTR collimation on the resolution of the measured electron beam divergence. The angular distributions of the DTR density for the indicated divergence $\psi_{0}=\sqrt{\psi_{0 \perp}^{2}+\psi_{0 \|}^{2}}$ are shown in Fig. 5 for $\psi_{0 \perp}=\psi_{0 \|}$. 


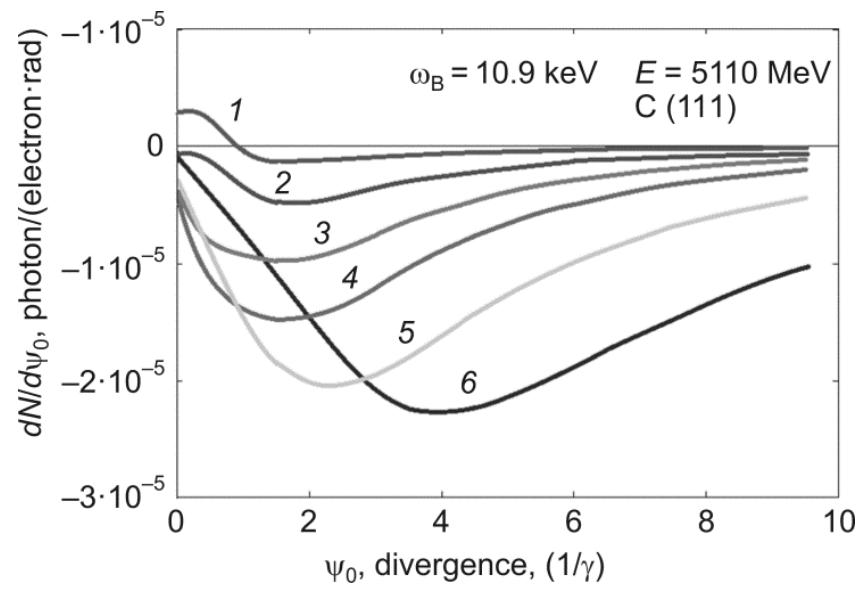

Fig. 4. Dependence of the DTR photon yield derivative $d N_{\mathrm{ph}} / d \psi_{0}$ on the electron beam divergence $\left(\gamma=10^{4}\right)$ for collimation angles $\theta_{c \|}=\theta_{c \perp}=0.5 \gamma^{-1}$ (curve 1$), \quad 1 \gamma^{-1}$ (curve 2$), 1.5 \gamma^{-1}$ (curve 3$), 2 \gamma^{-1}$ (curve 4$), 3 \gamma^{-1}$ (curve 5$)$, and $5 \gamma^{-1}$ (curve 6).

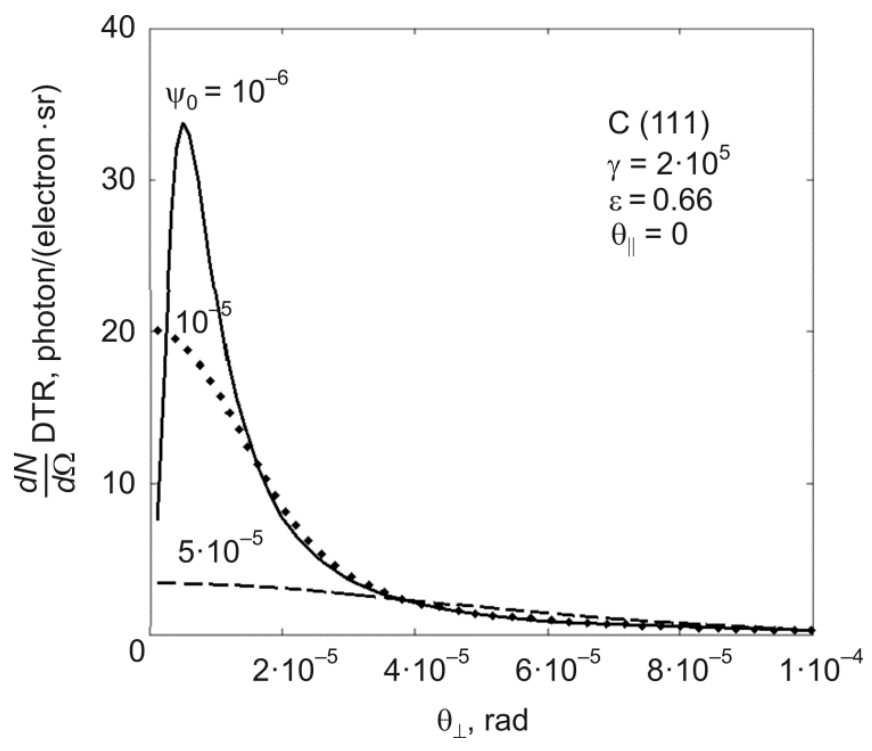

Fig. 5. Angular distribution of the DTR density for the indicated values of the electron beam divergence.

Thus, we can conclude that the yield of DTR photons emitted by the ultrahigh-energy electron beam traversing the thin single crystal target into a preset solid angle can be used for indicating the beam divergence. For example, from Fig. 4 it can be seen that for the divergence parameter lying in the range $1 / \gamma<\psi_{0}<10 / \gamma$, the collimation angle $\left(\theta_{c \|}=\theta_{c \perp}=5 / \gamma\right)$ can be used effectively without changing. At the second step, to refine the obtained divergence parameters, the collimation angle should be changed to a more optimal angle, and calculations should be repeated. The 
two-parametrical angular distributions with $\psi_{0 \|} \neq \psi_{0 \perp}$ can be indicated using different collimation angles $\theta_{c \|} \neq \theta_{c \perp}$ and a two-dimensional Gaussian function for averaging of the angular DTR density:

$$
f(\psi)=\frac{1}{\pi \psi_{0 \perp} \psi_{0 \|}} e^{-\left(\frac{\psi_{\perp}^{2}}{\psi_{0 \perp}^{2}}+\frac{\psi_{\|}^{2}}{\psi_{0 \|}^{2}}\right)} .
$$

To obtain an unambiguous solution of this problem, at least two independent measurements of the DTR photon yield must be performed, for example, using two slit collimators located perpendicularly each other.

In this case, two photon yields should be calculated for indicating the divergence parameters $\psi_{0 \perp}$ and $\psi_{0 \|}$ :

$$
\begin{gathered}
\left\langle N_{\text {DTR }}\left(\psi_{0 \perp}, \psi_{0 \|}\right)\right\rangle_{c \perp}=\frac{1}{\pi \psi_{0 \perp} \psi_{0 \|}} \int_{-\infty}^{\infty} \int_{-\infty}^{\infty} \int_{-\theta_{c \perp}}^{\theta_{c \perp}} \int_{-\infty}^{\infty} \sum_{s=1}^{2} \frac{d N_{\text {DTR }}^{(s)}}{d \Omega}\left(\psi_{\perp}, \psi_{\|}, \theta_{\perp}, \theta_{\|}\right) e^{-\left(\frac{\psi_{\perp}^{2}}{\psi_{0 \perp}^{2}}+\frac{\psi_{\|}^{2}}{\psi_{0 \|}^{2}}\right)} d \psi_{\perp} d \psi_{\|} d \theta_{\perp} d \theta_{\|}, \\
\left\langle N_{\text {DTR }}\left(\psi_{0 \perp}, \psi_{0 \|}\right)\right\rangle_{c \|}=\frac{1}{\pi \psi_{0 \perp} \psi_{0 \|}} \int_{-\infty}^{\infty} \int_{-\infty}^{\infty} \int_{-\infty}^{\infty} \int_{-\theta_{c \|}}^{\theta_{c \|}} \sum_{s=1}^{2}\left(\frac{d N_{\text {DTR }}^{(s)}}{d \Omega}\left(\psi_{\perp}, \psi_{\|}, \theta_{\perp}, \theta_{\|}\right)\right) e^{-\left(\frac{\psi_{\perp}^{2}}{\left.\psi_{0 \perp}^{2}+\frac{\psi_{\|}^{2}}{\psi_{0 \|}^{2}}\right)} d \psi_{\perp} d \psi_{\|} d \theta_{\perp} d \theta_{\|} \cdot\right.}
\end{gathered}
$$

The goal function can be constructed in the form

$$
F\left(\psi_{0 \perp}, \psi_{0 \|}\right)=\left(\left\langle N_{\mathrm{DTR}}\left(\psi_{0 \perp}, \psi_{0 \|}\right)\right\rangle_{c \perp}-N_{\mathrm{DTR}}^{\text {measured }}{ }_{c \perp}\right)^{2}+\left(\left\langle N_{\mathrm{DTR}}\left(\psi_{0 \perp}, \psi_{0 \|}\right)\right\rangle_{c \|}-N_{\mathrm{DTR}}^{\text {measured }}{ }_{c \|}\right)^{2} .
$$

The electron beam divergence parameters $\psi_{0 \perp}^{*}$ and $\psi_{0 \|}^{*}$ are determined from the condition of minimization of the goal function $F\left(\psi_{0 \perp}^{*}, \psi_{0 \|}^{*}\right)=\min \left(F\left(\psi_{0 \perp}, \psi_{0 \|}\right)\right)$ using one of the existing methods of minimization of a two-dimensional function. The plot of the goal function is shown in Fig. 6. The characteristic width of the angular DTR distribution generated by a single electron is equal to $\Delta \theta_{\mathrm{DTR}}=1 / \gamma$, where $\gamma$ is the relativistic factor of the emitting electron.

To obtain the resolution of the beam divergence close to the optimal one, the angular width of the slit collimator must be chosen of the order of $\theta_{c \perp}=\theta_{c \|} \approx 1 / \gamma$ for $\psi_{0 \perp} \approx \psi_{0 \|} \leq 1 / \gamma$, of the order of $\theta_{c \perp} \approx \psi_{0 \perp}$ for $\psi_{0 \perp}>1 / \gamma$, and $\theta_{c \|} \approx \psi_{0 \|}$ for $\psi_{0 \|}>1 / \gamma$. The accuracy of the solution depends on the statistical error $\Delta N_{\mathrm{ph}}^{\text {measure }} \approx t \sqrt{N_{\mathrm{ph}}^{\text {measure }}}$ of measuring the number of photons $N_{\mathrm{ph}}^{\text {measure }}$, where the coefficient $t$ defines the confidence interval (we used $t=3$ for a probability of $99.73 \%$ ). For example, if we require that the accuracy (the relative error) was $\delta_{N} \leq 0.01$, then the number of the recorded photons must be $N_{\mathrm{ph}}^{\text {measure }} \geq \frac{t^{2}}{\delta_{N}^{2}}=\frac{9}{0.0001}=9 \cdot 10^{4}$, which is quite real for the ILC accelerator under development in which $2.6 \cdot 10^{13}$ electrons will be used in one acceleration cycle. Indeed, as can be seen from Fig. 2, the number of DTR photons emitted by a single electron for the examined target will be of the order of $1 \cdot 10^{-8}$, and the corresponding number of photons emitted by the electron beam and equal to $2.6 \cdot 10^{13} \cdot 10^{-8}=2.6 \cdot 10^{5}$ will be sufficient for measuring the DTR photon yield with a required accuracy. The DTR intensity can be increased by increasing the thickness of the crystal target in the range satisfying to the conditions of minimal influence of the measurement process on the measured parameters of the electron beam and by increasing the asymmetry parameter $\varepsilon$ (see formula (11)). 


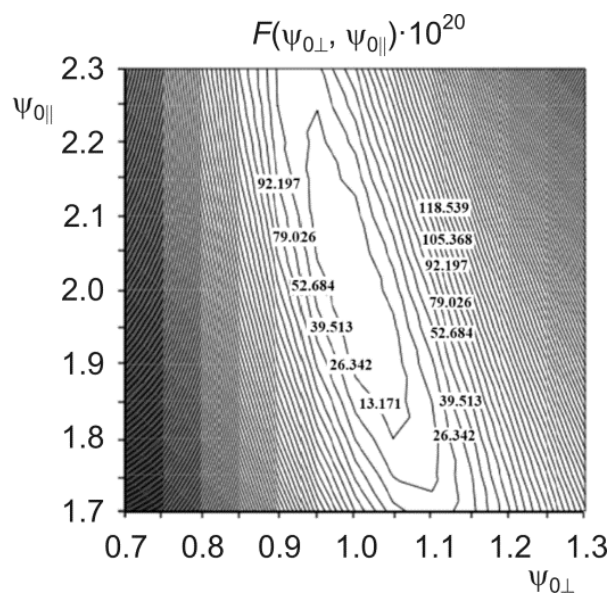

Fig. 6. Goal function (14) for determining the divergence parameters $\psi_{0 \perp}^{*}$ and $\psi_{0 \|}^{*}$ from the "measured" numbers of DTR photons $N_{\text {DTR }}^{\text {measured }}{ }_{c \perp}=6.942 \cdot 10^{-9}$ and $N_{\text {DTR }}^{\text {measured }}{ }_{c \|}=9.932 \cdot 10^{-9}$ (preliminary calculated from formulas (13) for $\psi_{0 \perp}=1 / \gamma$ and $\left.\psi_{0 \|}=2 / \gamma\right)$. The parameters $\psi_{0 \perp}$ and $\psi_{0 \|}$ are expressed in units of $1 / \gamma \mathrm{rad}$. The minimum value of the goal function was $F(1 / \gamma, 2 / \gamma)=0$.

Among other parameters used as experimental data containing errors are the photon collimator angles $\theta_{c \perp}$ and $\theta_{c \|}$. Thus, the accuracy of determining the electron beam divergence can be expressed in terms of the accuracy of the parameters $\theta_{c \perp}, \theta_{c \|}$, and $N_{\text {measured }}^{\text {ph }}$. We assume that calculation errors are absent. The errors of the absolute differences between the calculated and measured numbers of photons in the first and second terms of the goal function given by Eq. (14) can be written as follows:

$$
\begin{aligned}
& \Delta N_{c \|}\left(\psi_{0 \perp}, \psi_{0 \|}\right)=\Delta N_{\text {DTR }}^{\text {measured }}{ }_{c \|}+\frac{1}{\pi\left(\psi_{0 \perp}, \psi_{0 \|}\right)} \int_{-\infty}^{\infty} \int_{-\infty}^{\infty} \int_{-\infty}^{\infty} \sum_{s=1}^{2} \frac{d N_{\text {DTR }}^{(s)}}{d \Omega}\left(\psi_{\perp}, \psi_{\|}, \theta_{\perp}, \theta_{c \|}\right) e^{-\left(\frac{\psi_{\perp}^{2}}{\psi_{0 \perp}^{2}}+\frac{\psi_{\|}^{2}}{\psi_{0 \|}^{2}}\right)} d \psi_{\perp} d \psi_{\|} d \theta_{\perp} 2 \Delta \theta_{c \|}, \\
& \Delta N_{c \perp}\left(\psi_{0 \perp}, \psi_{0 \|}\right)=\Delta N_{\text {DTR }}^{\text {measured }}{ }_{c \perp}+\frac{1}{\pi\left(\psi_{0 \perp}, \psi_{0 \|}\right)} \int_{-\infty}^{\infty} \int_{-\infty}^{\infty} \int_{-\infty}^{\infty} \sum_{-\infty=1}^{2} \frac{d N_{\text {DTR }}^{(s)}}{d \Omega}\left(\psi_{\perp}, \psi_{\|}, \theta_{c \perp}, \theta_{\|}\right) e^{-\left(\frac{\psi_{\perp}^{2}}{\psi_{0 \perp}^{2}}+\frac{\psi_{\|}^{2}}{\psi_{0 \|}^{2}}\right)} d \psi_{\perp} d \psi_{\|} d \theta_{\|} 2 \Delta \theta_{c \perp},
\end{aligned}
$$

where the second term on the right-hand side of each of these expressions describes the contribution of errors of the parameter $\theta_{c \|}$ or $\theta_{c \perp}$. The errors of the calculated divergence parameters $\psi_{0 \perp}^{*}$ and $\psi_{0 \|}^{*}$ are written in terms of differences between the results of solving the minimization problem for function (14) for the measured numbers of photons $N_{\text {DTR }}^{\text {measured }}{ }_{c \perp}, N_{\text {DTR }}^{\text {measured }}{ }_{c \|}$ and $N_{\text {DTR }}^{\text {measured }}{ }_{c \perp}+\Delta N_{c \perp}\left(\psi_{0 \perp}, \psi_{0 \|}\right), N_{\text {DTR }}^{\text {measured }}{ }_{c \|}+\Delta N_{c \|}\left(\psi_{0 \perp}, \psi_{0 \|}\right)$.

Our estimates showed that the relative errors of the parameters $\theta_{c \perp}, \theta_{c \|}$, and $N_{\mathrm{ph}}^{\text {measured }}$ introduced the relative errors of the same order of magnitude in the calculated parameters $\psi_{0 \perp}^{*}$ and $\psi_{0 \|}^{*}$. The parameters of the electron beam divergence calculated by minimization of goal function (14) by the Hooke-Jeeves method are given in Table 1. The 
TABLE 1. Values of the Divergence Parameters $\psi_{0 \perp}$ and $\psi_{0 \|}$

\begin{tabular}{|c|c|c|c|c|}
\hline \multicolumn{5}{|c|}{ "Experimental" values of the electron beam divergence parameters } \\
\hline \multicolumn{3}{|c|}{$\psi_{0 \perp}^{(\exp )}=5 \cdot 10^{-6} \mathrm{rad}=1 / \gamma$} & \multicolumn{2}{|c|}{$\psi_{0 \|}^{(\exp )}=1 \cdot 10^{-5}=2 / \gamma$} \\
\hline \multicolumn{2}{|c|}{$N_{\text {DTR }}^{\text {measured }}{ }_{c \perp}=6.942 \cdot 10^{-9}$} & & \multicolumn{2}{|c|}{$N_{\text {DTR }}^{\text {measured }}{ }_{c \|}=9.932 \cdot 10^{-9}$} \\
\hline \multicolumn{5}{|c|}{ Calculated values } \\
\hline Iteration number $(i)$ & $\gamma \cdot \psi_{0 \perp}{ }^{(i)}$ & $\gamma \cdot \psi_{0 \|}{ }^{(i)}$ & $\gamma \cdot \Delta \psi_{0 \perp}{ }^{(i)}$ & $\gamma \cdot \Delta \psi_{0 \|}{ }^{(i)}$ \\
\hline 0 & 0.1 & 0.1 & 0.4 & 0.4 \\
\hline 5 & 0.9 & 1.7 & 0.1 & 0.05 \\
\hline 10 & 1.05 & 1.875 & $6.25 \cdot 10^{-3}$ & 0.0125 \\
\hline 15 & 1.00625 & 1.975 & $3.125 \cdot 10^{-3}$ & $6.25 \cdot 10^{-3}$ \\
\hline 20 & 1.0015625 & 1.996875 & $3.9063 \cdot 10^{-4}$ & $1.5625 \cdot 10^{-3}$ \\
\hline 25 & 1.0001953 & 1.9992187 & $9.7656 \cdot 10^{-5}$ & $1.953125 \cdot 10^{-4}$ \\
\hline
\end{tabular}

Note. Here the quantities calculated in the process of fitting to their "experimental" values are designated as follows: $\left(\psi_{0 \perp}\right)^{(\exp )}=1 / \gamma$ and $\left(\psi_{0 \|}\right)^{(\exp )}=2 / \gamma$ are calculated using the computational program for finding a minimum of the goal function by the Hook-Jeeves minimization method; $\psi_{0 \perp}{ }^{(0)}=\psi_{0 \perp}{ }^{(0)}=0.1 / \gamma$ is the initial value of the parameters; $\Delta \psi_{0 \perp}{ }^{(0)}=\Delta \psi_{0 \|}{ }^{(0)}=0.2 / \gamma$ is the initial increment of the parameters $\left(\max \left(\Delta \psi_{0 \perp}, \Delta \psi_{0 \perp}\right) \geq \varepsilon \varepsilon\right) ; \varepsilon \varepsilon=10^{-4}(1 / \gamma)$ is the required accuracy of the approximation $\left(\max \left(\Delta \psi_{0 \perp}, \Delta \psi_{0 \perp}\right) \geq \varepsilon \varepsilon\right) ; F\left(\psi_{0 \perp}, \psi_{0 \|}\right)$ is the goal function; $\gamma=2 \cdot 10^{5}$ is the relativistic factor of electron. The target represented (111)-oriented single-crystal diamond with the thickness $L_{t}=5 \cdot 10^{-5} \mathrm{~cm}$.

form of goal function (14) used to simulate the process of indicating the electron beam divergence parameters is shown in Fig. 6. The clearly pronounced minimum corresponding to the sought-after divergence parameters can be seen.

For the same conditions as in Table 1, except the parameter $N_{\mathrm{DTR}}^{\text {measured }}{ }_{c \perp}$ whose value was increased by $1 \%$, we obtained $\psi_{0 \perp}^{*}=1.0144531 / \gamma$, that is, by $1.4 \%$ more than in Table $1\left(\psi_{0 \perp}=1.000195 / \gamma\right)$, and $\psi_{0 \|}^{*}=1.97109$, that is, by $1.4 \%$ less than in Table $1\left(\psi_{0 \|}=1.999219 / \gamma\right)$.

\section{GOAL FUNCTION BASED ON THE MEASURED ANGULAR DTR DISTRIBUTION}

Virtual collimators formed for the measured angular distribution of DTR photons can be used as slit collimators to measure the parameters of electron beam divergence. This case can be realized with a two-dimensional matrix of photodetectors used to record the distribution. Then one measurement ensures two values of the DTR photon yield. The feasibility of application of the angular DTR photon distribution in calculations allows the goal function to be optimized by organization of virtual collimators within the limits of the recorded angular radiation distribution. The virtual slit collimators based on the matrix of the measured angular DTR distribution will contain a certain common area of their crossing that can be excluded from consideration, thereby increasing the sensitivity of the method of minimization of goal function (14). In particular, calculations of the photon yields indicating the divergence parameters $\psi_{0 \perp}$ and $\psi_{0 \|}$ can be performed using modified expressions (13) and (14), respectively: 


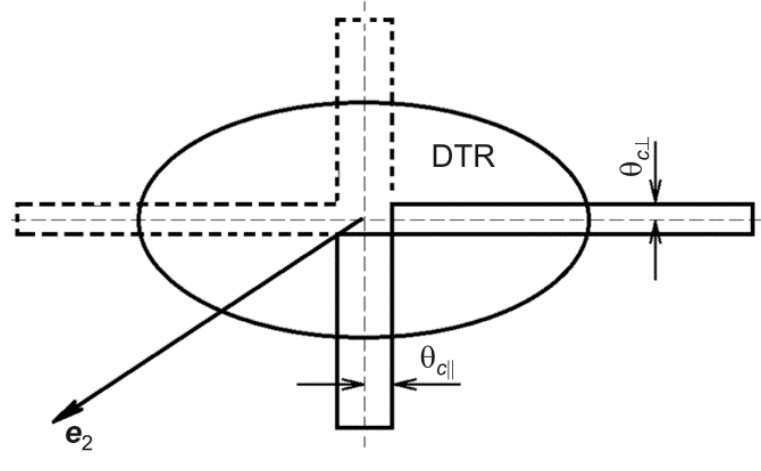

Fig. 7

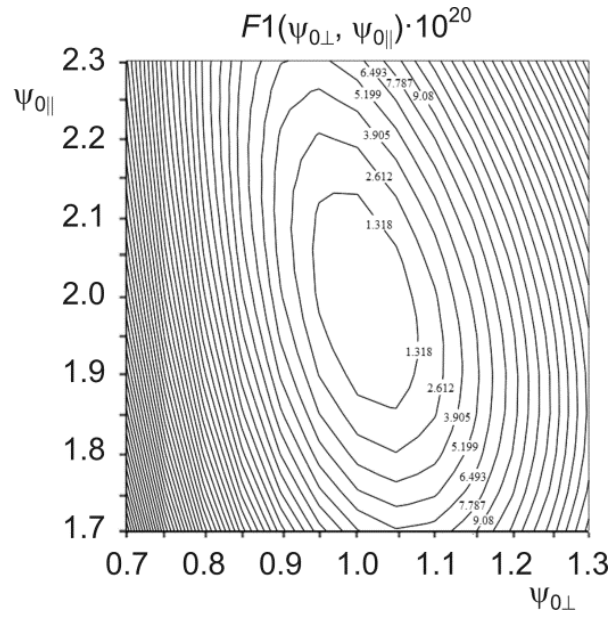

Fig. 8

Fig. 7. Formation of the virtual slit collimators $\theta_{c \perp}$ and $\theta_{c \|}$ on the matrix of the recorded angular DTR distribution by summation over the pixel values and comparison (using the goal function $F 1$ ) with the calculated photon numbers.

Fig. 8. Goal function (17) for determining the electron beam divergence parameters $\psi_{0 \perp}^{*}$ and $\psi_{0\|\|}^{*}$ from the "measured" numbers of DTR photons $N_{\mathrm{DTR}}^{\text {measured }}{ }_{c \perp}=1.559 \cdot 10^{-9}$ and $N_{\text {DTR }}^{\text {measured }}{ }_{c \|}=3.054 \cdot 10^{-9}$ (preliminary calculated by Eq. (16) for $\psi_{0 \perp}=1 / \gamma$ and $\left.\psi_{0 \|}=2 / \gamma\right)$. The parameters $\psi_{0 \perp}$ and $\psi_{0 \| \mid}$ are expressed in units of $1 / \gamma \mathrm{rad}$. The value of the minimum goal function was $F 1(1 / \gamma, 2 / \gamma)=0$.

$$
\begin{aligned}
& \left\langle N 1_{\mathrm{DTR}}\left(\psi_{0 \perp}, \psi_{0 \|}\right)\right\rangle_{c \perp}=\frac{1}{\pi \psi_{0 \perp} \psi_{0 \|}} \int_{-\infty}^{\infty} \int_{-\infty}^{\infty} \int_{-\theta_{c \perp}}^{\theta_{c \perp}} \int_{\theta_{c \mid}}^{\infty} \sum_{s=1}^{2} \frac{d N_{\mathrm{DTR}}^{(s)}}{d \Omega}\left(\psi_{\perp}, \psi_{\|}, \theta_{\perp}, \theta_{\|}\right) \cdot e^{-\left(\frac{\psi_{\perp}^{2}}{\psi_{0 \perp}^{2}}+\frac{\psi_{\|}^{2}}{\psi_{0 \|}^{2}}\right)} d \psi_{\perp} d \psi_{\|} d \theta_{\perp} d \theta_{\|}, \\
& \left\langle N 1_{\mathrm{DTR}}\left(\psi_{0 \perp}, \psi_{0 \|}\right)\right\rangle_{c \|}=\frac{1}{\pi \psi_{0 \perp} \psi_{0 \|}} \int_{-\infty}^{\infty} \int_{-\infty}^{\infty} \int_{\theta_{c \|}}^{\infty} \int_{\theta_{c \|}}^{\theta_{c \|}} \sum_{s=1}^{2}\left(\frac{d N_{\mathrm{DTR}}^{(s)}}{d \Omega}\left(\psi_{\perp}, \psi_{\|}, \theta_{\perp}, \theta_{\|}\right)\right) e^{-\left(\frac{\psi_{\perp}^{2}}{\psi_{0 \perp}^{2}}+\frac{\psi_{\|}^{2}}{\psi_{0 \|}^{2}}\right)} d \psi_{\perp} d \psi_{\|} d \theta_{\perp} d \theta_{\|}
\end{aligned}
$$

The corresponding goal function can be constructed in the form

$$
F 1\left(\psi_{0 \perp}, \psi_{0 \|}\right)=\left(\left\langle N 1_{\text {DTR }}\left(\psi_{0 \perp}, \psi_{0 \|}\right)\right\rangle_{c \perp}-N_{\text {DTR }}^{\text {measured }}{ }_{c \perp}\right)^{2}+\left(\left\langle N 1_{\text {DTR }}\left(\psi_{0 \perp}, \psi_{0 \|}\right)\right\rangle_{c \|}-N_{\text {DTR }}^{\text {measured }}{ }_{c \|}\right)^{2} .
$$

The scheme of the collimator formed in accordance with the integral limits in Eq. (16) is shown in Fig. 7.

Figure 8 shows the goal function based on the expression for the photon yield determined by the slit "collimators" with excluded common area around the $\boldsymbol{e}_{2}$ axis (see Fig. 1). It can be seen that the goal function 
TABLE 2. Results of Fitting to Sought-after Values of the Divergence Parameters of the Goal Function $F 1\left(\psi_{0 \perp}, \psi_{0 \|}\right)$

"Experimental" values of the electron beam divergence parameters

\begin{tabular}{c|c}
\hline$\psi_{0 \perp}^{(\text {exp) }}=5 \cdot 10^{-6} \mathrm{rad}=1 / \gamma$ & $\psi_{0 \|}^{(\mathrm{exp})}=1 \cdot 10^{-5}=2 / \gamma$ \\
\hline$N_{\text {DTR }}^{\text {measured }}{ }_{c \perp}=1.559 \cdot 10^{-9}$ & $N_{\text {DTR }}^{\text {measured }}{ }_{c \|}=3.054 \cdot 10^{-9}$ \\
\hline
\end{tabular}

\begin{tabular}{c|c|c|c|c}
\hline \multicolumn{5}{|c}{ Calculated values } \\
\hline Iteration number $(i)$ & $\gamma \cdot \psi_{0 \perp}{ }^{(i)}$ & $\gamma \cdot \psi_{0 \|}{ }^{(i)}$ & $\gamma \cdot \Delta \psi_{0 \perp}{ }^{(i)}$ & $\gamma \cdot \Delta \psi_{0 \|}{ }^{(i)}$ \\
\hline 0 & 0.1 & 0.1 & 0.1 & 0.1 \\
\hline 5 & 0.9 & 1.1 & 0.1 & 0.1 \\
\hline 10 & 1.1 & 1.9 & $6.25 \cdot 10^{-3}$ & 0.05 \\
\hline 11 & 1.05 & 2 & $6.245 \cdot 10^{-3}$ & 0.05 \\
\hline 12 & 1.05 & 2 & $3.125 \cdot 10^{-3}$ & 0.025 \\
\hline 13 & 1.05 & 1.95 & $3.125 \cdot 10^{-3}$ & 0.025 \\
\hline 14 & 1.0375 & 2 & $3.125 \cdot 10^{-3}$ & 0.025 \\
\hline 15 & 1.01875 & 1.95 & $3.125 \cdot 10^{-3}$ & 0.025 \\
\hline 25 & 0.999999 & 2 & $1.907 \cdot 10^{-7}$ & $3.815 \cdot 10^{-7}$ \\
\hline
\end{tabular}

Note. The same as in Table 1 , but for the goal function $F 1\left(\psi_{0 \perp}, \psi_{0 \|}\right)$. Here the accuracy parameter $\varepsilon \varepsilon=10^{-7}(1 / \gamma)$ and the initial increment of the parameters $\Delta \psi_{0 \perp}{ }^{(0)}=\Delta \psi_{0 \|}{ }^{(0)}=0.1 / \gamma$; $\gamma=2 \cdot 10^{5}$ is the Lorentz factor of relativistic electron. The target represented the (111)-oriented single crystal of diamond with the thickness $L_{t}=5 \cdot 10^{-5} \mathrm{~cm}$.

$F 1\left(\psi_{0 \perp}, \psi_{0 \|}\right)$ has a more clearly pronounced minimum corresponding to the sought-after electron beam divergence parameters.

Results illustrating fitting to the sought-after values of the divergence parameters obtained with the goal function in the form $F 1\left(\psi_{0 \perp}, \psi_{0 \|}\right)$ are presented in Table 2 .

An important point of the proposed method is the application of the slit collimators with the parameters $\theta_{c \perp}=\psi_{0 \perp}$ and $\theta_{0 \|}=\psi_{0 \|}$ close to optimal ones that ensures the high resolution of the method of determining the electron beam divergence. It can be seen that under these conditions, the collimator parameters are expressed through unknown values of the divergence parameters of "measured" DTR. In this regard, instead of the "measured" values $N_{\text {DTR }}^{\text {measured }}{ }_{c \perp}$ and $N_{\text {DTR }}^{\text {measured }}{ }_{c \|}$ presented below, we must use the new goal function based on the functions $N_{\text {DTR }}^{\text {measured }}{ }_{c \perp}\left(\psi_{0 \perp}, \psi_{0 \|}\right)$ and $N_{\text {DTR }}^{\text {measured }}{ }_{c \|}\left(\psi_{0 \perp}, \psi_{0 \|}\right)$ :

$$
\begin{gathered}
F 2\left(\psi_{0 \perp}, \psi_{0 \|}\right)=\left(\left\langle N 2_{\mathrm{DTR}}\left(\psi_{0 \perp}, \psi_{0 \|}\right)\right\rangle_{c \perp}-N_{\mathrm{DTR}}^{\text {measured }}{ }_{c \perp}\left(\psi_{0 \perp}, \psi_{0 \|}\right)\right)^{2} \\
+\left(\left\langle N 2_{\mathrm{DTR}}\left(\psi_{0 \perp}, \psi_{0 \|}\right)\right\rangle_{c \|}-N_{\mathrm{DTR}}^{\text {measured }}\left(\psi_{0 \perp}, \psi_{0 \|}\right)_{c \|}\right)^{2}
\end{gathered}
$$

where 


$$
\begin{aligned}
& N 2_{\mathrm{DTR}}\left(\psi_{0 \perp}, \psi_{0 \|}\right)_{c \perp}=\left\langle N_{\mathrm{DTR}}\left(\psi_{0 \perp}, \psi_{0 \|}, \psi_{0 \perp}, \psi_{0 \|}\right)\right\rangle_{c \perp}, \\
& N 2_{\mathrm{DTR}}\left(\psi_{0 \perp}, \psi_{0 \|}\right)_{c \|}=\left\langle N_{\mathrm{DTR}}\left(\psi_{0 \perp}, \psi_{0 \|}, \psi_{0 \perp}, \psi_{0 \|}\right)\right\rangle_{c \|}, \\
& N_{\mathrm{DTR}}^{\text {measured }}\left(\psi_{0 \perp}, \psi_{0 \|}\right)_{c \perp}=\left\langle N_{\mathrm{DTR}}\left(\psi_{0 \perp}^{\text {measured }}, \psi_{0 \|}^{\text {measured }}, \psi_{0 \perp}, \psi_{0 \|}\right)\right\rangle_{c \perp}, \\
& N_{\text {DTR }}^{\text {measured }}\left(\psi_{0 \perp}, \psi_{0 \|}\right)_{c \|}=\left\langle N_{\text {DTR }}\left(\psi_{0 \perp}^{\text {measured }}, \psi_{0 \|}^{\text {measured }}, \psi_{0 \perp}, \psi_{0 \|}\right)\right\rangle_{c \|}, \\
& \left\langle N_{\mathrm{DTR}}\left(\psi_{0 \perp}, \psi_{0 \|}, \theta_{c \perp}, \theta_{c \|}\right)\right\rangle_{c \perp}=\frac{1}{\pi \psi_{0 \perp} \psi_{0 \|}} \int_{-\infty}^{\infty} \int_{-\infty}^{\infty} \int_{-\theta_{c \perp}}^{\theta_{c \perp}} \int_{\theta_{c \mid}}^{\infty} \sum_{s=1}^{2} \frac{d N_{\mathrm{DTR}}^{(s)}}{d \Omega}\left(\psi_{\perp}, \psi_{\|}, \theta_{\perp}, \theta_{\|}\right) e^{-\left(\frac{\psi_{\perp}^{2}}{\psi_{0 \perp}^{2}}+\frac{\psi_{\|}^{2}}{\psi_{0 \|}^{2}}\right)} d \psi_{\perp} d \psi_{\|} d \theta_{\perp} d \theta_{\|},
\end{aligned}
$$

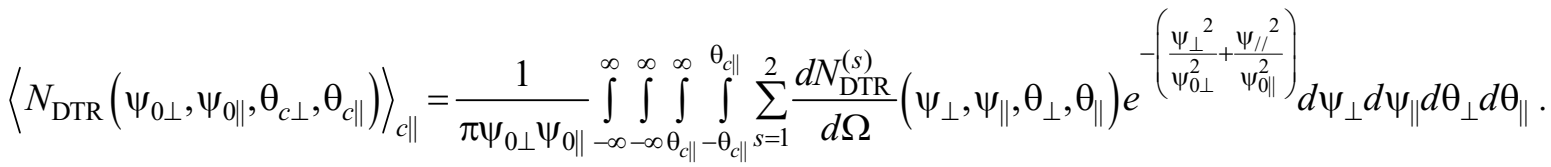

It is obvious that in practice, the electron beam divergence parameters will be determined for a discrete distribution of the angular DTR density over pixels of the recording matrix. In this case, the measured collimated DTR yield can be represented in terms of the goal function $F 2\left(\psi_{0 \perp}, \psi_{0 \|}\right)$ using interpolated functions. On the other hand, it is possible to use a simpler method of minimization of the goal function, since the number of pixels for the angular DTR distribution will be limited. The independence of the total DTR yield on the electron beam divergence (see curve 8 in Fig. 2) can be used to solve the problem of indicating the divergence of the measured collimated DTR yield normalized by the electron beam intensity.

\section{ASSESSMENT OF A REAL SITUATION}

At the beginning, we estimate the conditions of experimental implementation of the proposed methods of indicating the electron beam divergence based on consideration of the typical beam parameters of the ILC collider: the electron energy of $250 \mathrm{GeV}$; the electron beam cross section $S_{\text {beam }} \simeq 5 \cdot 100 \mathrm{~nm}^{2}$; and the beam divergence $\psi_{0} \simeq 1 / \gamma$. To evaluate the feasibility of measuring the angular DTR distribution, we must know the actual parameters of the existing x-ray detector matrices. If the spatial resolution of the matrix does not exceed $5 \mu \mathrm{m}$ and the number of pixels is about 10000, the transverse size of the recorded DTR flux will be $D_{L_{\mathrm{ph}}} \simeq 10^{-3} \mathrm{~m}$. For the parameters of the electron beam divergence $\psi_{0 \perp} \simeq \psi_{0 \|} \simeq 1 / \gamma$, the distance from the target to the detector can be defined as $L_{\mathrm{ph}} \simeq D_{L_{\mathrm{ph}}} \cdot \psi_{0} \simeq D_{L_{\mathrm{ph}}} \cdot \gamma \simeq 5 \cdot 10^{2} \mathrm{~m}$. This is the quite real distance for the x-ray propagation without losses through the photon channel in vacuum $P \leq 10^{-6}$ Torr. 


\section{CONCLUSIONS}

Diffracted transition radiation emitted by the relativistic electron beam traversing a thin single-crystal plate in the Laue scattering geometry has been studied. For the first time, the expression describing the angular density of DTR generated when the electron path length in the target is significantly shorter than the extinction length has been derived. The structure of the given expression shows the kinematic character of the DTR process under these conditions. The expression describing the average number of DTR photons emitted by the diverging beam of relativistic electrons with ultrahigh energies traversing a thin single-crystal plate within a given solid angle in the direction of Bragg's scattering was obtained. The numerical calculations were performed demonstrating the dependence of the collimated DTR photon yield on the beam divergence.

To determine the divergence parameters of the electron beam with the angular distribution described by a twodimensional normal distribution with different variances along the coordinates, we used expressions for the DTR photon yields for the slit collimators located perpendicularly each other. The goal function used for calculation of the divergence parameters of the electron beam was constructed and the expression for estimation of the error in determining the beam divergence parameters was derived. Model calculations of the divergence parameters of the electron beam based on the "measured" yield of DTR photons for the slit collimators were carried out. The efficiency of the algorithm for estimation of the beam divergence parameters based on the Hook-Jeeves minimization method was demonstrated. The expressions obtained can be successfully used as a basis for the development of methods of measuring the beam divergence of ultrahigh-energy relativistic electrons based on the angular DTR distribution.

\section{REFERENCES}

1. ILC Technical Design Report (2013); http://ww2.linearcollider.org/ILC/Publications/Technical-Design-Report.

2. M. Aicheler, ed., A Multi-TeV linear collider based on CLIC technology: CLIC Conceptual Design Report, CERN (2012).

3. Y. Takabayashi, Phys. Lett. A, 376, 2408 (2012).

4. Y. Takabayashi and K. Sumitani, Phys. Lett. A, 377, 2577 (2013).

5. B. N. Kalinin, A. P. Potylitsin, V. A. Verzilov, et al., Nucl. Instrum. Methods Phys. Res. A, 350, 601 (1994).

6. A. Gogolev, A. Potylitsyn, and G. Kube, J. Phys. Conf. Series, 357, 012018 (2011).

7. A. Caticha, Phys. Rev. A, 40, 4322 (1989).

8. V. Baryshevsky, Nucl. Instrum. Methods A, 122, 13 (1997).

9. X. Artru and P. Rullhusen, Nucl. Instrum. Methods B, 145, 1 (1998).

10. N. Nasonov, Phys. Lett. A, 246, 148 (1998).

11. S. V. Blazhevich, G. A. Grazhdankin, R. A. Zagorodnyuk, and A. V. Noskov, Nucl. Instrum. Methods Phys. Res. B, 355, 170-174 (2015). 